\title{
Detection of Ciprofloxacin-Resistant, $\beta$-Lactamase-Producing Neisseria meningitidis Serogroup Y Isolates — United States, 2019-2020
}

\begin{abstract}
Lucy A. McNamara, PhD ${ }^{1}$; Caelin Potts, $\mathrm{PhD}^{1}$; Amy E. Blain, MPH${ }^{1}$; Adam C. Retchless, $\mathrm{PhD}^{1}$; Natashia Reese, $\mathrm{MS}^{2}$; Stephanie Swint, MS ${ }^{2}$; David Lonsway, MMSc ${ }^{2}$; Maria Karlsson, $\mathrm{PhD}^{2}$; Kristy Lunquest, ScM ${ }^{3}$; John J. Sweitzer, ScM ${ }^{3}$; Xin Wang, $\mathrm{PhD}^{1}$; Susan $\mathrm{Hariri}$, PhD ${ }^{1}$; LeAnne M. Fox, $\mathrm{MD}^{1}$; Antimicrobial-Resistant Neisseria meningitidis Team
\end{abstract}

Meningococcal disease is a sudden-onset, life-threatening illness caused by the bacterium Neisseria meningitidis. Prompt empiric antibiotic treatment can reduce morbidity and mortality among patients, and antibiotic prophylaxis can prevent secondary disease in close contacts. Historically, N. meningitidis isolates in the United States have largely been susceptible to the antibiotics recommended for treatment and prophylaxis, including penicillin and ciprofloxacin. This report describes detection of penicillin-resistant and ciprofloxacin-resistant $N$. meningitidis serogroup $\mathrm{Y}(\mathrm{NmY})$ isolates in the United States. NmY isolates containing a bla $\mathrm{ROB}-1 \beta$-lactamase enzyme gene conferring resistance to penicillins (1) were recovered from 33 cases reported during 2013-2020. Isolates from 11 of these cases, reported during 2019-2020, harbored a ciprofloxacin resistance-associated mutation in a chromosomal gene $(\mathrm{g} y \mathrm{r} A)$. Cases were reported from 12 geographically disparate states; a majority of cases ( 22 of $33,67 \%)$ occurred in Hispanic persons. These cases represent a substantial increase in penicillin-resistant and ciprofloxacin-resistant meningococci in the United States since 2013. Ceftriaxone and cefotaxime, the recommended first-line agents for empiric bacterial meningitis treatment, can continue to be used for treatment, but health care providers should ascertain susceptibility of meningococcal isolates to penicillin before switching to penicillin or ampicillin. Ongoing monitoring for antimicrobial resistance among meningococcal isolates and prophylaxis failures will be important to inform treatment and prophylaxis recommendations.

Meningococcal disease is a severe illness with a sudden onset and $10 \%-15 \%$ case-fatality rate. The disease is typically treated empirically with cefotaxime or ceftriaxone, which can be changed to penicillin or ampicillin once $N$. meningitidis is confirmed as the causative pathogen (2). Because close contacts of meningococcal disease patients have an elevated risk for disease (3), they are recommended to receive antibiotic prophylaxis with ciprofloxacin, rifampin, or ceftriaxone as soon as a suspected meningococcal disease case is identified (4).

Resistance to the antibiotics used for meningococcal treatment and prophylaxis has been rare among $N$. meningitidis isolates in the United States (5). Although intermediate penicillin susceptibility is common among meningococci, the clinical relevance of this finding is unclear. Penicillin resistance in
$N$. meningitidis attributable either to $\beta$-lactamase production or to other mechanisms is rare $(5,6)$. Resistance to ciprofloxacin is also uncommon in the United States with only one identified cluster of three ciprofloxacin-resistant cases during 2007-2008 and infrequent sporadic cases $(5,7,8)$. Because $N$. meningitidis is typically susceptible to clinically relevant antibiotics in the United States, antimicrobial susceptibility testing is not routinely performed on meningococcal isolates (9).

In January 2020, an $\mathrm{NmY}$ isolate that produced a $\beta$-lactamase and was resistant to penicillin and ciprofloxacin was cultured from a meningococcal disease case in a Maryland resident (Gillian Taormina, Benjamin Hanisch, Children's National Hospital, Washington, DC, personal communication; 2020). When a second case of infection with a $\beta$-lactamaseproducing, ciprofloxacin-resistant $\mathrm{NmY}$ isolate was reported by the Maryland Department of Health in February 2020, a systematic analysis of $N$. meningitidis isolates in the United States was conducted to determine whether this resistance pattern was more widespread.

Isolates from meningococcal disease cases are submitted to CDC approximately every 6 months by health departments from all states, Washington, D.C., and New York City. For this investigation, $\mathrm{CDC}$ requested that health departments submit to CDC all NmY isolates from cases during 2019-2020 that had not yet been submitted. The request was made through CDC's Epi-X (https://emergency.cdc.gov/epix/index.asp) secure communications network for public health officials with follow-up by e-mail to each state health department. Isolates, or confirmation that no additional isolates were available, were received from 24 state health departments and the District of Columbia.

Whole genome sequencing (WGS) was performed on all available meningococcal isolates from U.S. invasive meningococcal disease cases that occurred during 2011-2020. Sequencing data were analyzed to assess the presence of the bla $a_{\mathrm{ROB}-1} \beta$-lactamase gene and mutations associated with ciprofloxacin resistance. Isolates with both a $\beta$-lactamase gene and ciprofloxacin resistance-associated mutations underwent reference antimicrobial susceptibility testing at $\mathrm{CDC}$ to assess $\beta$-lactamase activity and susceptibility to penicillin, ciprofloxacin, and third-generation cephalosporins. State health departments provided supplementary epidemiologic data from 
case investigation records for cases with isolates containing a $\beta$-lactamase gene.

A total of 2,097 N. meningitidis isolates underwent WGS; 372 of these isolates were NmY. Analysis of WGS data identified 11 serogroup $\mathrm{Y}$ isolates that contained a bla $\mathrm{ROB}-1$ $\beta$-lactamase gene and a T91I gyrA mutation associated with resistance to ciprofloxacin. An additional 22 isolates contained bla $a_{\mathrm{ROB}-1}$ but did not have mutations associated with ciprofloxacin resistance; 21 of these isolates were serogroup Y while one was nongroupable (NG). All $33 \beta$-lactamase-containing isolates were in clonal complex 23 (CC23); 30, including all 11 with ciprofloxacin resistance mutations, were sequence type (ST)-3587; two were ST-15379; and one was ST-13034. The 33 isolates were from cases occurring in 12 states during 20132020 (Figure 1) (Figure 2). Antimicrobial susceptibility testing was conducted on the 11 isolates with ciprofloxacin resistance mutations; all were confirmed to produce a $\beta$-lactamase and to be resistant to penicillin and ciprofloxacin but susceptible to third-generation cephalosporins, rifampin, and azithromycin.

A majority of the meningococcal disease cases caused by isolates containing bla ROB-1 $_{1}$ occurred in young children and older adults (Table). Notably, although there were no known epidemiologic links among the 33 cases, 22 (67\%) occurred in Hispanic persons, including eight of the 11 cases with ciprofloxacin-resistant isolates. Only one case was fatal (casefatality rate $=3.0 \%$ ).

\section{Discussion}

This evaluation identified a novel, emerging strain of penicillin-resistant and ciprofloxacin-resistant, $\beta$-lactamaseproducing $N$. meningitidis in the United States. The detection of geographically diverse cases with penicillin-resistant and ciprofloxacin-resistant $\mathrm{NmY}$ isolates has implications for treatment and prophylaxis of meningococcal disease in the United States.

Ceftriaxone and cefotaxime are the recommended firstline agents for empiric bacterial meningitis treatment and can continue to be used (2). However, given the number of $\beta$-lactamase-producing isolates detected and availability of other effective treatment options, health care providers in the United States should ascertain susceptibility of meningococcal isolates to penicillin before using penicillin or ampicillin for treatment.

Ongoing monitoring for antimicrobial resistance among meningococcal isolates and for prophylaxis failures will be important to inform whether changes to meningococcal disease prophylaxis guidance is needed. A 2-day course of rifampin or a single injection of ceftriaxone are recommended alternatives to ciprofloxacin for prophylaxis of contacts of persons with meningococcal disease (4) but are logistically more challenging to administer. A single dose of azithromycin can also be used for prophylaxis in communities where ciprofloxacin-resistant meningococci have been detected; however, there is only a

FIGURE 1. Clonal complex 23 Neisseria meningitidis isolates $(\mathrm{N}=33)$ with a bla $a_{\mathrm{ROB}-1} \beta$-lactamase enzyme gene* alone or in combination with a ciprofloxacin resistance-associated mutation (cipro-R), by quarter - United States, 2013-2020

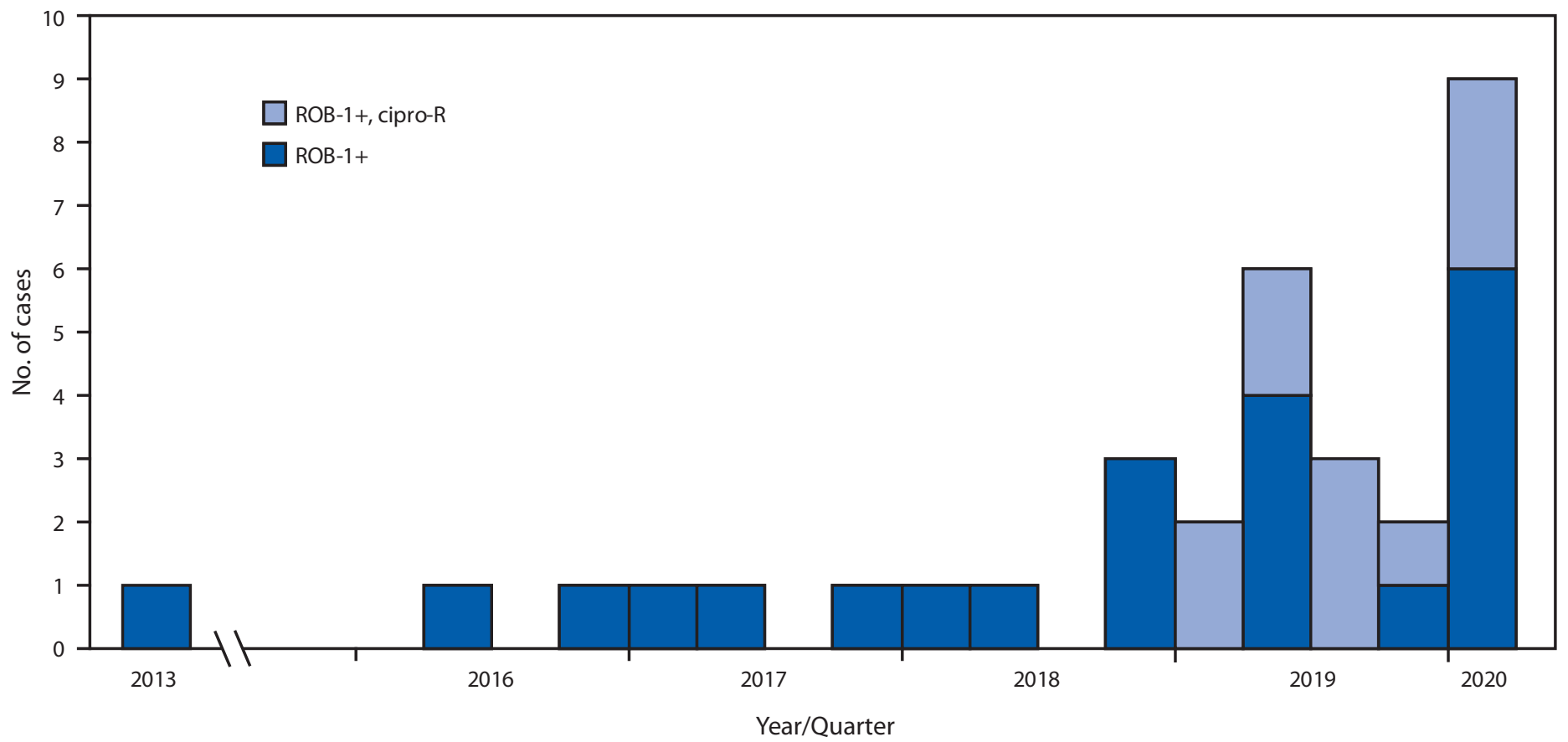

* Conferring resistance to penicillins. 
FIGURE 2. Meningococcal disease cases with clonal complex 23 Neisseria meningitidis isolates $(\mathrm{N}=33)$ with a bla $a_{\mathrm{ROB}-1} \beta$-lactamase enzyme gene* alone or in combination with a ciprofloxacin resistance-associated mutation, by state - United States, 2013-2020

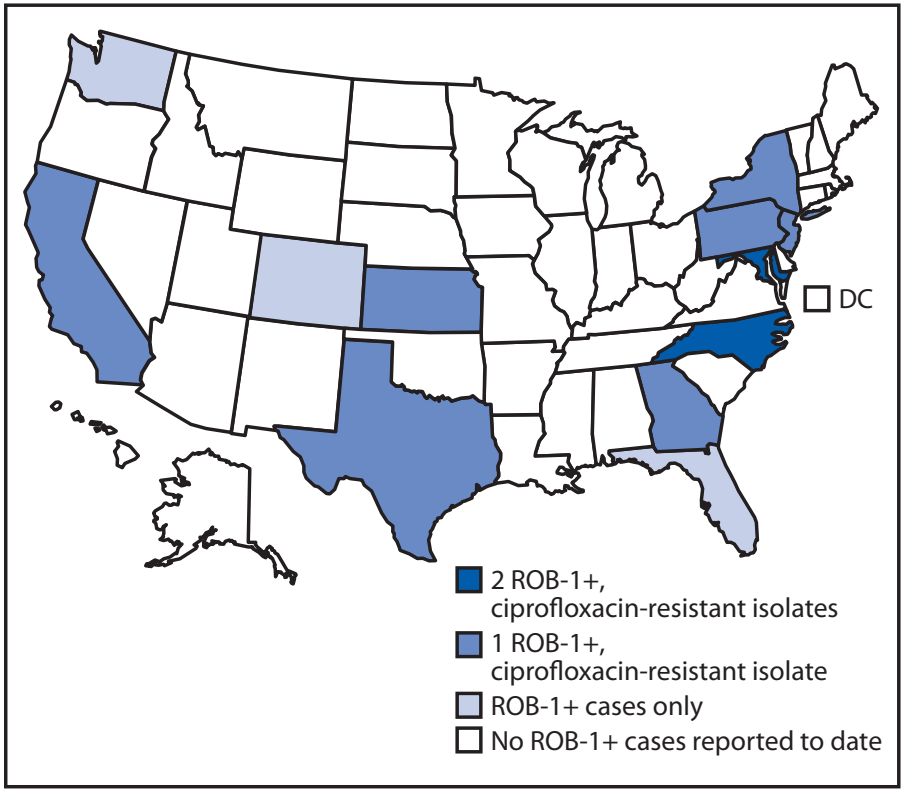

Abbreviation: $\mathrm{DC}=$ District of Columbia.

* Conferring resistance to penicillins.

single published study demonstrating effectiveness of azithromycin for clearing meningococcal carriage (4).

It is unknown how widely the $\beta$-lactamase-positive, ciprofloxacin-resistant $\mathrm{NmY}$ strain detected in the United States might be circulating in other countries. Penicillin-resistant and ciprofloxacin-resistant $\mathrm{NmY}$ isolates were detected in El Salvador during 2017-2019, but similar cases have not been reported elsewhere. Single $\mathrm{NmY}$ isolates positive for bla $\mathrm{ROB}-1$ $\beta$-lactamase but susceptible to ciprofloxacin have also been reported from Canada $(\sigma)$ and France $(10)$. The potential circulation of penicillin-resistant or ciprofloxacin-resistant meningococci in other countries merits further investigation.

These findings show that penicillin-resistant and ciprofloxacin-resistant meningococci are now present in the United States; however, the complete geographic and temporal distribution of these resistant meningococci is unclear, because not all U.S. meningococcal disease cases have isolates available for WGS or antimicrobial susceptibility testing. In 2017 and 2018, CDC received isolates for only $72 \%$ and $78 \%$ of U.S. meningococcal disease cases, respectively*; submission of isolates from meningococcal disease cases that occurred during 2019-2020 is ongoing. The coronavirus disease 2019

\footnotetext{
*https://www.cdc.gov/meningococcal/downloads/NCIRD-EMS-Report-2017. pdf and https://www.cdc.gov/meningococcal/downloads/NCIRD-EMSReport-2018.pdf.
}

TABLE. Epidemiologic and clinical characteristics of meningococcal disease cases caused by bla $a_{\mathrm{ROB}-1}$-containing Neisseria meningitidis, United States, 2013-2020

\begin{tabular}{|c|c|c|c|}
\hline \multirow[b]{2}{*}{ Characteristic } & \multicolumn{3}{|c|}{ No. (\%) } \\
\hline & All ROB- $1+^{*}$ & ROB-1+ only & $\begin{array}{l}\text { ROB-1+, } \\
\text { ciprofloxacin- } \\
\text { resistant }\end{array}$ \\
\hline Total & 33 & 22 & 11 \\
\hline \multicolumn{4}{|l|}{ Age group (yrs) } \\
\hline$<1$ & $6(18)$ & $3(14)$ & $3(27)$ \\
\hline $1-10$ & $4(12)$ & $3(14)$ & $1(9)$ \\
\hline $11-23$ & $2(6.1)$ & $1(4.5)$ & $1(9)$ \\
\hline $24-44$ & $6(18)$ & $4(18)$ & $2(18)$ \\
\hline $45-64$ & $10(30)$ & $7(32)$ & $3(27)$ \\
\hline$\geq 65$ & $5(15)$ & $4(18)$ & $1(9)$ \\
\hline \multicolumn{4}{|l|}{ Sex } \\
\hline Male & $18(54)$ & $9(41)$ & $9(82)$ \\
\hline Female & $15(45)$ & $13(59)$ & $2(18)$ \\
\hline \multicolumn{4}{|l|}{ Race/Ethnicity } \\
\hline Hispanic & $22(67)$ & $14(64)$ & $8(73)$ \\
\hline White, non-Hispanic & $4(12)$ & $4(18)$ & $0(-)$ \\
\hline Black $^{\dagger}$ & $6(18)$ & $3(14)$ & $3(27)$ \\
\hline Unknown & $1(3.0)$ & $1(4.5)$ & $0(-)$ \\
\hline \multicolumn{4}{|l|}{ Outcome } \\
\hline Survived & 32 (97) & $21(95)$ & $11(100)$ \\
\hline Died & $1(3.0)$ & $1(4.5)$ & $0(一)$ \\
\hline
\end{tabular}

* ROB- $1+$ is a $\beta$-lactamase enzyme gene that confers resistance to penicillins.

$\dagger$ Ethnicity of two black patients was not reported. Remaining black patients were non-Hispanic.

(COVID-19) pandemic has limited the submission of meningococcal isolates and collection of epidemiologic data and precluded phenotypic antimicrobial susceptibility testing on all isolates containing a $\beta$-lactamase gene.

To facilitate ongoing monitoring of antimicrobial resistance, state and territorial health departments are asked to continue submitting all meningococcal isolates to CDC for antimicrobial susceptibility testing and WGS and to report any suspected meningococcal treatment or prophylaxis failures. In states that have experienced meningococcal disease cases caused by ciprofloxacin-resistant strains during the past 1-2 years, clinicians and public health staff members should consider antimicrobial susceptibility testing on meningococcal isolates to inform prophylaxis decisions. ${ }^{\dagger}$ Antimicrobial susceptibility testing should not delay the initiation of prophylaxis. Jurisdictions with capacity for $\beta$-lactamase screening or WGS might also wish to assess $\beta$-lactamase production or presence of $\beta$-lactamase genes and ciprofloxacin resistance-associated mutations. States conducting their own antimicrobial susceptibility testing,

\footnotetext{
$\dagger$ Rigorous protection from droplets and aerosols, including use of a biosafety cabinet, is required when microbiologic procedures are performed on N. meningitidis isolates (https://www.cdc.gov/meningitis/lab-manual/chpt11antimicrobial-suscept-testing.html). Microbiologists who are routinely exposed to $N$. meningitidis isolates should receive meningococcal vaccines in accordance with current Advisory Committee on Immunization Practices recommendations (https://www.cdc.gov/vaccines/hcp/acip-recs/vacc-specific/mening.html).
} 


\section{Summary}

What is already known about this topic?

Most Neisseria meningitidis isolates in the United States have been susceptible to antibiotics recommended for treatment and prophylaxis.

What is added by this report?

During 2019-2020, 11 meningococcal isolates from U.S. patients had isolates containing a bla $a_{\mathrm{ROB}-1} \beta$-lactamase gene associated with penicillin resistance and mutations associated with ciprofloxacin resistance. An additional 22 cases reported during 2013-2020 contained bla $a_{\mathrm{ROB}-1}$ but did not have mutations associated with ciprofloxacin resistance.

What are the implications for public health practice?

Ceftriaxone and cefotaxime can continue to be used for empiric bacterial meningitis treatment; meningococcal isolate susceptibility to penicillin should be determined before switching to penicillin or ampicillin. Prophylaxis failures and antimicrobial resistance among meningococcal isolates should be monitored to inform meningococcal prophylaxis recommendations.

$\beta$-lactamase screening, or WGS are requested to share results and sequences with CDC. For cases with isolates determined to be $\beta$-lactamase screen-positive or ciprofloxacin-resistant, jurisdictions are requested to obtain and submit a supplementary case report form (https://www.cdc.gov/meningococcal/ surveillance/index.html).

\section{Acknowledgments}

Veronica Pinell-McNamara, Keegan Rudmann, Bacterial Meningitis Laboratory team members, CDC; Amanda Metz, Emily Spence-Davizon, Karen Xavier, Colorado Department of Public Health and Environment; Scott Pritchard, Florida Department of Health; Chelsea Raybern, Kansas Department of Health and Environment; David Torpey, Maryland Public Health Laboratory staff members, Maryland Department of Health; Mink Antwi, Paula Del Rosso, Marie Dorisinville, Don Weiss, Jessica Sell, Jennifer Rakeman-Cagno, Ulrike Siemetzki-Kapoor, New York City Department of Health and Mental Hygiene; Lisa Dettinger, Venkata Vapchedu, Pennsylvania Department of Health Bureau of Laboratories; John Faherty Philadelphia Department of Health; Greg Leos, Texas Department of State Health Services; vaccinepreventable diseases surveillance staff members of the Connecticut, Hawaii, Illinois, Indiana, Louisiana, Massachusetts, Michigan, Minnesota, Mississippi, Montana, Nevada, Oklahoma, South Carolina, Tennessee, Utah, West Virginia, and Wisconsin state health departments.

\section{Antimicrobial-Resistant Neisseria meningitidis Team}

Nirmala Dhungana, California Department of Public Health; Ryan Gabrio-Brannon, California Department of Public Health; Jennifer Kyle, California Department of Public Health; Brittany Martin, California Department of Public Health; Meghan Barnes, Colorado Department of Public Health and Environment; Ashley Moore, Georgia Department of Public Health; Susan Hannagan, New Jersey Department of Health; Page Keating, New York City Department of Health and Mental Hygiene; Sandy Li, New York City Department of Health and Mental Hygiene; Justin Albertson, North Carolina Department of Health and Human Services; Wayne Fleming, Bureau of Epidemiology, Pennsylvania Department of Health; Perrianne Lurie, Bureau of Epidemiology, Pennsylvania Department of Health; Christina Russell, Bureau of Laboratories, Pennsylvania Department of Health; Kara Reid, Philadelphia Department of Health; Kelsey Sanders, Texas Department of State Health Services; Chas DeBolt, Washington State Department of Health; Nicholas Graff, Washington State Department of Health; Esther Lam, Washington State Department of Health; Benjamin Hanisch, Children's National Hospital, Washington, DC; Gillian Taormina, Children's National Hospital, Washington, DC.

Corresponding author: Lucy A. McNamara, xdf4@cdc.gov, 404-218-3111.

${ }^{1}$ Division of Bacterial Diseases, National Center for Immunization and Respiratory Diseases, CDC; ${ }^{2}$ Division of Healthcare Quality Promotion, National Center for Emerging and Zoonotic Infectious Diseases, CDC; ${ }^{3}$ Maryland Department of Health, Baltimore.

All authors have completed and submitted the International Committee of Medical Journal Editors form for disclosure of potential conflicts of interest. No potential conflicts of interest were disclosed.

\section{References}

1. San Millan A, Escudero JA, Catalan A, et al. $\beta$-lactam resistance in Haemophilus parasuis is mediated by plasmid $\mathrm{pB} 1000$ bearing bla $\mathrm{ROB}-1$. Antimicrob Agents Chemother 2007;51:2260-4. https://doi. org/10.1128/AAC.00242-07

2. Meningococcal infections. In: Kimberlin DW, Brady MT, Jackson MA, Long SS, eds. Red book: 2018-2021 report of the committee on infectious diseases, 31st edition. Itasca, IL: American Academy of Pediatrics; 2018. https://redbook.solutions.aap.org/chapter.aspx?sectio nid $=189640131 \&$ bookid $=2205$

3. Cohn AC, MacNeil JR, Clark TA, et al.; CDC. Prevention and control of meningococcal disease: recommendations of the Advisory Committee on Immunization Practices (ACIP). MMWR Recomm Rep 2013;62(No. RR-2).

4. McNamara LA, Blain A. Meningococcal disease. In: Roush SW, Baldy LM, Hall MAK, eds. Manual for the surveillance of vaccine-preventable diseases. Atlanta, GA: US Department of Health and Human Services, CDC; 2019. https://www.cdc.gov/vaccines/pubs/surv-manual/chpt08mening.html

5. Harcourt BH, Anderson RD, Wu HM, et al. Population-based surveillance of Neisseria meningitidis antimicrobial resistance in the United States. Open Forum Infect Dis 2015;2:ofv117. https://doi. org/10.1093/ofid/ofv117

6. Tsang RSW, Ahmad T, Jamieson FB, Tyrrell GJ. WGS analysis of a penicillin-resistant Neisseria meningitidis strain containing a chromosomal ROB-1 $\beta$-lactamase gene. J Antimicrob Chemother 2019;74:22-8. 
7. Wu HM, Harcourt BH, Hatcher CP, et al. Emergence of ciprofloxacinresistant Neisseria meningitidis in North America. N Engl J Med 2009;360:886-92. https://doi.org/10.1056/NEJMoa0806414

8. CDC. Emergence of fluoroquinolone-resistant Neisseria meningitidisMinnesota and North Dakota, 2007-2008. MMWR Morb Mortal Wkly Rep 2008;57:173-5.
9. Blain AE, Mandal S, Wu H, et al. Penicillin use in meningococcal disease management: Active Bacterial Core Surveillance sites, 2009. Open Forum Infect Dis 2016;3:ofw152. https://doi.org/10.1093/ofid/ofw152 10. Hong E, Deghmane AE, Taha MK. Acquisition of beta-lactamase by Neisseria meningitidis through possible horizontal gene transfer. Antimicrob Agents Chemother 2018;62:e00831-00918. https://doi. org/10.1128/AAC.00831-18 